\title{
IKEA, un modèle territorial « en kit » : stratégies d'implantation et pouvoirs locaux
}

IKEA, a pre-assembled territorial model: location strategies and local authorities IKEA, ein Modell als Fertigbausatz: Siedlungsstrategien und lokale Mächte

Jonathan Musereau et Solène Gaudin

\section{(2) OpenEdition}

Journals

Édition électronique

URL : http://journals.openedition.org/rge/3056

DOI : $10.4000 /$ rge.3056

ISSN : $2108-6478$

Éditeur

Association des géographes de l'Est

Édition imprimée

Date de publication : 31 décembre 2010

ISSN : 0035-3213

\section{Référence électronique}

Jonathan Musereau et Solène Gaudin, «IKEA, un modèle territorial « en kit » : stratégies d'implantation et pouvoirs locaux », Revue Géographique de l'Est [En ligne], vol. 50 / 3-4 | 2010, mis en ligne le 04 novembre 2011, consulté le 10 décembre 2020. URL : http://journals.openedition.org/rge/3056 ; DOI : https://doi.org/10.4000/rge.3056

Ce document a été généré automatiquement le 10 décembre 2020.

Tous droits réservés 


\section{IKEA, un modèle territorial « en kit » : stratégies d'implantation et pouvoirs locaux}

IKEA, a pre-assembled territorial model: location strategies and local authorities IKEA, ein Modell als Fertigbausatz: Siedlungsstrategien und lokale Mächte

Jonathan Musereau et Solène Gaudin

\section{Introduction}

Leader mondial de la distribution anomale ${ }^{1}$, IKEA imprègne le tissu économique et social des territoires qu'il investit. Ses stratégies en matière d'implantation et d'interaction avec les acteurs publics cristallisent un certain nombre d'interrogations récurrentes dans littérature qui lui est consacrée (Bailly \& Caudron, 2006 ; Déjardins, 2002). Qu'apporte un IKEA au territoire local ? La standardisation marketing impose-telle une normalisation territoriale ? Quel rôle joue la firme auprès des acteurs locaux ? En somme, quelles sont les implications d'un groupe à la stratégie mondiale et mondialisée dans les perspectives d'un développement local. Cette contribution s'inscrit dans le champ de l'étude des dynamiques territoriales et d'une géographie des acteurs situés (Lajarge \& Gumuchain et al., 2003) dans un contexte de mondialisation. Il s'agit ici de présenter les liens existants et les capacités d'interaction entre une Firme Trans-Nationale (FTN) et les différentes échelles territoriales de décision, ceci au travers d'un panorama des stratégies internes et externes d'IKEA. On se propose d'approfondir les effets d'ancrage et d'appel d'une FTN dans le jeu des méso-échelles de la décision (et de l'ingénierie) territoriale.

A l'échelle infranationale, IKEA semble prendre le contre-pied d'une localisation liée à un rapprochement des centres majeurs d'impulsion (Badré \& Ferrand, 2001). Les sites d'IKEA ne reflètent pas les contours classiques de la hiérarchisation établie des territoires (Gaudin \& Musereau, 2009). Au-delà de cette approche rationnelle et économique, d'autres éléments prennent une part croissante dans les choix de 
localisation de la firme. L'étude de l'espace européen démontre qu'IKEA s'appuie fréquemment sur le soutien des élus et fonctionne comme une structure tremplin pour les territoires périphériques. Mais elle révèle également des enjeux contradictoires en termes d'emploi et de développement économique, particulièrement à travers l'analyse des antagonismes associés aux récents projets de magasins sur le territoire français.

\section{IKEA, mode d'emploi. Une inscription globale dans des espaces d'affinités}

«Un Ikea sur le territoire de la commune, c'est la gloire!» s'exclame un conseiller municipal de Stockport en Grande-Bretagne ${ }^{2}$.A chaque implantation de l'enseigne suédoise correspond un véritable événement régional. Promoteur de décors de cinéma, de services de restauration, d'une voiture écologique - la LEKA - et même récemment d'une sitcom, IKEA est une firme polymorphe et rayonnante dont le succès ne se dément pas depuis 30 ans. Même les polémiques sociales et environnementales ne semblent pas écorner l'image jeune, chaleureuse et dynamique du géant de l'ameublement. En véritable promoteur de l'aménagement territorial, la firme ne se contente plus de s'appuyer sur les composantes et les potentialités des territoires, appréhendées comme simples « supports de facteur de localisation » (Storaï, 2003), elle agit avec et auprès des pouvoirs publics responsables. Ainsi, en Belgique, la loi de 2004 sur l'autorisation des implantations commerciales ne passe pas inaperçue. Couramment désignée sous l'appellation " loi IKEA », elle marque la simplification de l'octroi socioéconomique et la promotion de la libre entreprise sur le territoire belge. Accordant un rôle décisif à l'échelle communale, la loi IKEA souligne surtout le rôle croissant joué par les firmes sur et dans les politiques locales comme nationales ${ }^{3}$.

\section{A. « Un Ikea sinon rien ! » : Pouvoirs, décisions et séduction}

Les firmes multinationales sont des acteurs essentiels de l'économie et du pouvoir à l'échelle mondiale. Les théoriciens de l'Ecole de la Régulation (notamment: Boyer, 1996 ; Commaille \& Jobert, 1999 ; Laffont \& Tirole, 1993) ont montré combien, dès les années 1970, dans un système de libéralisation des structures économiques, les institutions et les Etats-nations ne s'effaçaient pas derrière les règles du libre échange mais participaient plus ou moins directement à conditionner les modalités des investissements privés. Dans un contexte de désengagement contractualisé et progressif de l'Etat (Epstein, 2009), la prééminence des acteurs économiques dans le jeu local de la gouvernance urbaine remet en cause l'appréciation dichotomique des enjeux de pouvoir au sein de l'action publique. L'imbrication des intérêts entre les parties révèle un processus de complexification et de pluralisation des stratégies (Pinson, 2010) et des systèmes d'acteurs ancrés dans des territoires. La logique d'attraction des IDE s'est donc largement substituée aux politiques étatiques de restriction-réglementation des implantations des firmes étrangères (Andreff, 1999). Pour une large part, ce sont désormais les choix stratégiques de localisation des firmes multinationales qui préfigurent la réalisation des nouveaux relais de la croissance, en tant qu'élément moteur de la rationalisation de l'économie et de l'agencement des territoires (Cohen \& Lorenzi, 2000). Ces évolutions se lisent dans l'économie mais aussi dans la vie politique des pays sensibles au contexte libéral. En 2004, au moment même où la loi irlandaise, à 
travers le Planing Board, restreint à $6000 \mathrm{~m}^{2}$ la superficie maximum des magasins de la grande distribution, IKEA projette une implantation à Dublin. A l'issue de plusieurs mois de négociations et face au refus de la firme de revoir ses plans, le Ministre de l'Environnement de l'époque décide de modifier la loi à l'avantage de l'enseigne suédoise faisant fi de l'opposition ouverte des plus petites structures commerciales déjà en place sur le territoire irlandais. Quand la firme ne parvient à trouver un compromis acceptable, elle n'hésite pas non plus à entamer des procédures judiciaires à l'encontre de ses détracteurs, y compris lorsque ceux-ci représentent les autorités locales, comme dans l'exemple du refus d'implantation d'un magasin près de Manchester, également en 2004. Suite à un recours en justice contre la décision du cabinet du Premier ministre refusant le projet d'implantation sur le site de Stockport et n'ayant pas obtenu satisfaction devant les tribunaux, la firme parviendra malgré tout à recevoir une autorisation de construction à Ashton-Under-Line, non loin du site initialement envisagé.

La coproduction entre des acteurs locaux, nationaux et internationaux dont résulte les nouveaux agencements des territoires amène à une réflexion élargie sur les déterminants de l'action publique et sur les modalités afférentes aux décisions prises par la FTN. Si les facteurs traditionnels de l'attractivité territoriale sont bien connus en matière d'accès au marché, de potentiel de développement et d'intégration économique (Porter, 1982 ; Lorenzi, 2004), le choix d'une implantation demeure toujours un enjeu stratégique, tant pour la firme que pour les territoires d'accueil. Les représentants des administrations étatiques conservent $a$ priori l'essentiel de leurs prérogatives puisqu'ils sont chargés de fixer les normes portant sur la gestion de ces territoires. Or, en matière de gouvernance urbaine et de régimes urbains, l'observation de la recomposition des jeux politiques (G. Pinson, 2010) s'articule autour du renouvellement des régulations de l'action publique. La relecture des territoires opérée par la firme ouvrirait-elle la voie à une légitimation de la régionalisation des modes de gouvernance, suggérant une redéfinition des périmètres de l'action publique?

\section{B. Les territoires d'accueil : Un modèle d'expansion non linéaire}

L'expansion d'IKEA est remarquable, tant au niveau de ses volumes de vente, du taux d'embauche ou de son extension géographique. En 2008, avec quatre cent dix millions de clients, le groupe suédois comptait 230 magasins implantés sur 4 continents et opérait dans 44 pays : l'Europe (28), l'Amérique du Nord (2), le Moyen-Orient (5) et l'Asie-Pacifique (8). Il n'en demeure pas moins de très fortes disparités dans le recouvrement régional : l'Allemagne détient le record de densité avec 38 magasins, la Suède en dénombre 14 et la Finlande voisine n'en compte que 2 quand la Chine vient d'inaugurer son cinquième magasin. Malgré une large dispersion géographique, le chiffre d'affaires de la firme est réalisé à $81 \%$ dans l'espace européen et atteint 20 milliards d'euros. On y conçoit et fait fabriquer des meubles dans le monde entier : $20 \%$ de la production est réalisée en Europe de l'Est, $24 \%$ en Asie (Vietnam, Philippines, Inde...). La logistique et les prix bas sont au cœur de la stratégie d'IKEA, dont l'argument commercial phare est de proposer à une large clientèle (même si la cible principale est plutôt jeune) des produits de qualité à prix réduit et dans un conditionnement permettant une flexibilité nouvelle pour la vente de biens anomaux de ce type. Pour mettre en œuvre sa vision des affaires, le groupe s'est doté d'une 
structure et d'un concept qui englobent toutes les étapes, de l'idée initiale du produit à son arrivée chez le consommateur.

«L'IKEA way " repose sur une dimension culturelle nationale promue en stratégie de marketing, comme l'illustre la diffusion des choix d'implantation de la firme à l'échelle du continent européen (figure 1). Le premier magasin a été ouvert en 1958, dans la ville d'Älmhult, siège de l'entreprise. Il faudra attendre 5 ans pour qu'un magasin s'implante en dehors de Suède, près d'Oslo, et 1973 pour voir un magasin IKEA hors de Scandinavie, à proximité de Zurich. Rapidement l'Allemagne concentre l'essentiel des installations nouvelles. Les implantations en France sont un peu plus tardives, seulement à partir de 1983. Leur répartition reflète les écarts structurels du pays avec, aux abords de la région parisienne, la plus forte concentration au monde de magasins. Si on observe plus finement les stratégies chrono-spatiales de la firme, on remarque que l'expansion d'IKEA ne reflète pas précisément les contours classiques de la hiérarchisation établie des territoires (Gaudin \& Musereau, 2009). L'enseigne bleu et jaune a par exemple investi le marché italien en ignorant sa capitale pendant plus d'une dizaine d'années. Plus généralement, le tissu de magasins en Europe met en exergue une sorte de clivage Est-Ouest, l'un étant particulièrement bien fourni et l'autre relativement délaissé. Cette opposition apparente montre la prédominance d'un double facteur de localisation, associant la densité de population et, dans une moindre mesure, le pouvoir d'achat.

Figure 1 : Expansion d'IKEA à travers l'Europe

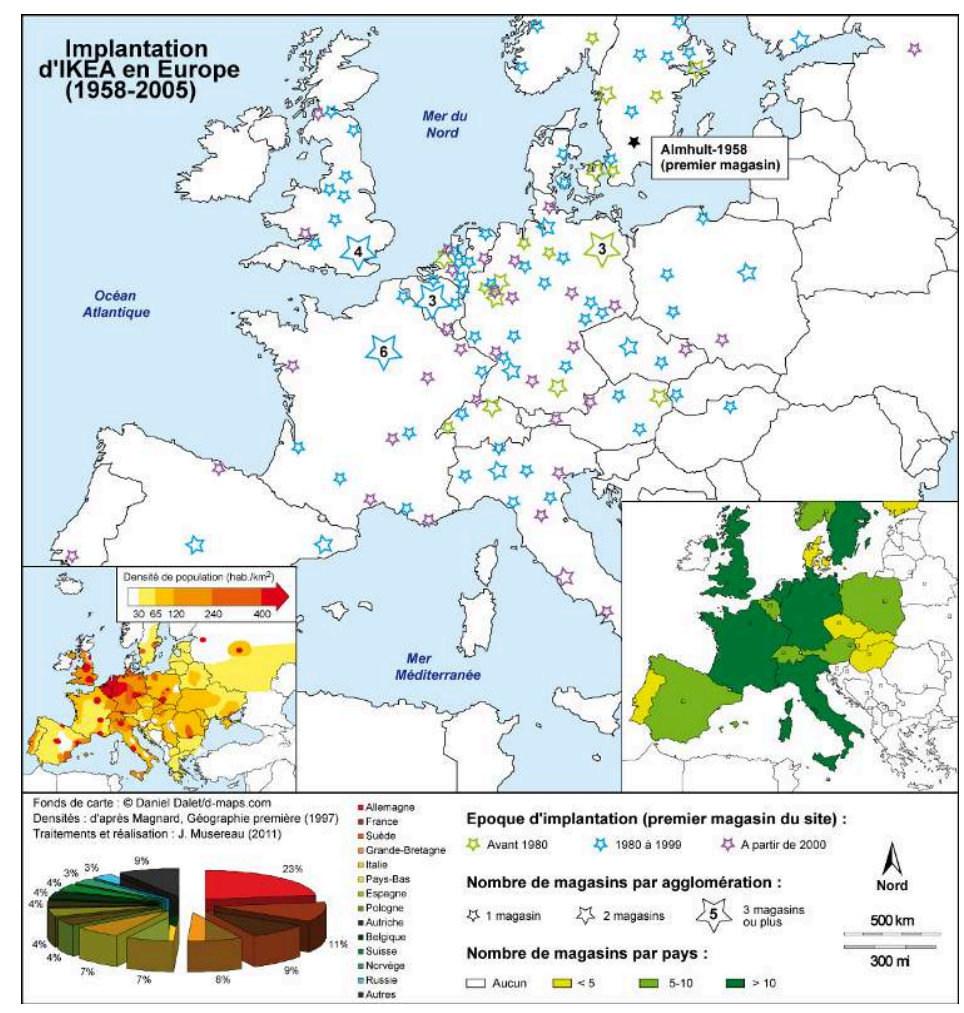

Au-delà des déterminants économiques traditionnels, cette situation traduit de fortes affinités culturelles dans une diffusion géographique qui n'est pas nécessairement proximale, hiérarchique, ni même mixte. Les dirigeants de l'entreprise expliquent leur positionnement par un souhait marqué d'intégration économique et territoriale dans 
les pays investis. IKEA a préféré retirer provisoirement ses projets d'implantation en Inde en raison d'une législation trop restrictive en matière de détention des capitaux et de développement stratégique global. Fort d'une stratégie commerciale hybride (Norman \& Ramirez, 1993), d'un concept marketing universel et d'une neutralité des produits proposés, le groupe suédois est pourtant actif en matière de prospection territoriale et de localisation stratégique. Après le groupe Auchan, IKEA est une des premières firmes d'envergure à avoir investi massivement sur le marché russe après l'effondrement soviétique. L'objectif était d'implanter un centre de production dans un pays low cost et de s'affranchir des droits de douane $(25 \%)$ sur les meubles importés en recourant au sourcing ${ }^{4}$. Le groupe souhaitait utiliser les capacités de production du pays pour approvisionner son réseau mondial de magasins. Avec une demande globale qui double tous les quatre ans, la sécurisation de l'approvisionnement est logiquement devenue un enjeu majeur pour IKEA. Dans ces conditions, un partenariat s'est imposé avec les acteurs locaux, la Russie détenant à elle seul un quart des réserves mondiales en bois. Pour permettre aux fournisseurs russes d'acheter l'équipement industriel nécessaire et ouvrir son unité de production, le groupe a du investir plus de 400 millions de dollars.

Même si la firme est résolument internationalisée et mondialisée, une importance prééminente de facteurs économiques traditionnels explique également les choix d'IKEA : les affinités culturelles, la proximité économique et managériale, la PPA mais aussi la matière première et de facto une dimension géostratégique et politique de premier plan. En conséquence, il existe bien une complexification de la lecture géostratégique de la firme concernant aussi bien ses filières d'approvisionnement que ses facteurs de distribution et d'implantation.

\section{Inscriptions et ancrages d'une FTN idéo-centrée}

Dans sa stratégie d'implantation, l'enseigne suédoise privilégie essentiellement deux critères intangibles : rassembler dans un périmètre accessible en une heure de voiture une densité d'au moins un million de personnes. Le résultat pour la Bretagne est éloquent (figure 2A). Revue et corrigée par IKEA, la structure du territoire régional se résumerait à une bipolarité autour des centres rennais et brestois, alors qu'une bande centrale serait exclue. A cette échelle, la corrélation avec les valeurs de densité est donc réduite. Si 8 des 10 communes les plus peuplées de la région sont situées sur le littoral, la convergence en direction du bord de mer n'entre pas en ligne de compte dans la stratégie de la firme. A l'échelle régionale, l'accessibilité aux bassins de peuplement majeurs devient donc un facteur prépondérant. On comprend alors pourquoi l'emprise des magasins est très souvent matérialisée par le tracé des principaux axes routiers (figure 2B). Les projections relatives au projet Wuxi (Chine) en constituent une parfaite illustration. La cartographie de l'aire de chalandise de ce futur complexe commercial suit fidèlement la structure urbaine « en doigts de gant ». Dans de très nombreux cas, les décisions stratégiques de la firme dépendent de fait d'un troisième facteur, celui des opportunités foncières et du souhait de se situer à proximité des principaux axes de communication. Grands consommateurs d'espace, les magasins IKEA nécessitent en effet de vastes superficies pour s'implanter : 5 à 6 hectares en moyenne d'un seul tenant, une surface standard proche de $30000 \mathrm{~m}^{2}$ pour le magasin et une surface de vente atteignant en moyenne près de $20000 \mathrm{~m}^{2}$. Les espaces convoités sont majoritairement les mêmes que ceux des complexes commerciaux situés dans la proche 
banlieue, parfois des zones déprimées ou laissées en friches car les prix du terrain y sont plus bas. Chez le géant suédois, la pondération des facteurs de décision stratégique se résumerait donc à un triptyque dont l'efficacité n'est plus à prouver (figure $2 \mathrm{C}$ ).

Figure 2 : La stratégie d'implantation des magasins IKEA

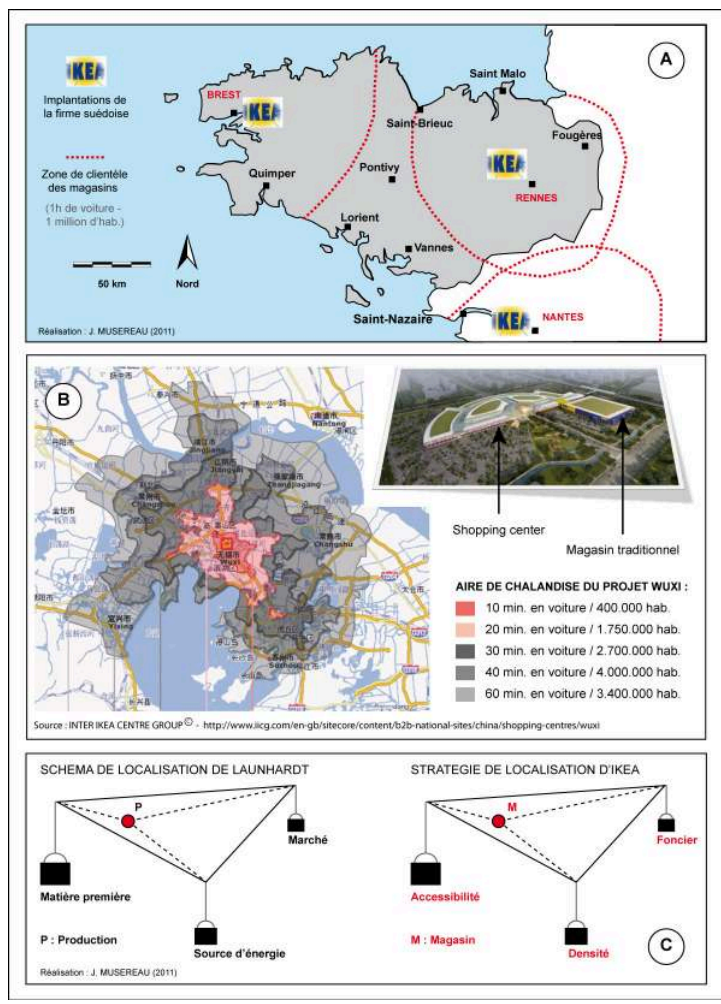

Très majoritairement, les magasins de l'enseigne se situent à l'extérieur des centresvilles, sur les marges ou en périphéries. Cette localisation préférentielle s'explique à la fois par les coûts du foncier et par l'attraction des axes de communication, qui ont la double fonction d'accessibilité et de visibilité du magasin. Quelques tentatives de magasins de plus petites tailles néanmoins ont vu le jour, sans succès. On peut citer ceux d'Ottawa (Canada), de Heerlen (Pays-Bas) ou de Manhattan (Etats-Unis). Ces tentatives demeurent infructueuses car le concept repose avant tout sur la dimension d'appel de l'infrastructure (la curieuse «boîte bleue») et sur des produits de consommation toujours identifiés comme relevant majoritairement de biens anomaux. En décembre 2007, le groupe suédois expérimente à Coventry (Royaume-Uni) le premier magasin conventionnel - mais réparti sur 7 étages - en plein cœur de la ville. Ce projet est rendu possible par le développement d'un ensemble commercial dont IKEA intègre et promeut la mise en place. Il faut préciser aussi l'intérêt du site à proximité de Birmingham, directement relié et accessible par l'autoroute en moins de vingt minutes. Ce concept est enfin une réponse face aux mesures mise en place par le gouvernement et les autorités locales pour limiter les vastes implantations commerciales à la périphérie des villes.

En conséquence il existe bien une complexification de la lecture géostratégique de la firme concernant aussi bien ses filières d'approvisionnement que ses facteurs de distribution et d'implantation. Le marché n'est pas le seul déterminant. Les effets 
transformationnels de l'inscription territoriale de la firme se lisent aussi aux échelles infranationales (Berthaud \& Rocca, 2000).

\section{Une insertion territoriale ambivalente}

Successivement capteurs, attracteurs et macro-régulateurs des tissus économiques territorialisés, les Etats agissent comme des éléments assurant le fonctionnement d'un système social dynamique, un faisceau de relations économiques et politiques. A cette échelle, l'alternative n'est plus seulement réduite au choix entre la compensation, la compétition ou la protection (Rogowski, 1989). La véritable lutte des territoires s'est reportée à une échelle infra-territoriale qui impose une lecture et un redécoupage des échelles de la gouvernance sur le mode d'une régulation négociée.

\section{A. Un espace en tractation pour une gouvernance pluraliste et territorialisée}

Le Groupe IKEA appartient à une fondation basée aux Pays-Bas, la Stichting Ingka, qui détient Ingka Holding B.V., la société mère de toutes les sociétés, du groupe industriel Swedwood aux filiales de distribution nationale (figure 3). Ingka Holding B.V. a la responsabilité des activités du Groupe et de la direction générale du Groupe IKEA. Inter IKEA Systems B.V. est le propriétaire du concept et de la marque utilisés par l'ensemble des magasins IKEA. La plupart des magasins sont détenus par le Groupe IKEA, au travers d'accords de franchise. Chaque magasin du groupe reverse ainsi une franchise de $3 \%$ à Inter IKEA System, elle-même associée à Ingka Holding respectivement implantées au Luxembourg et aux Pays-Bas. IKEA fonctionne par une intégration totale de sa logistique et contrôle toutes les étapes, de la conception à la commercialisation des produits. Études de marché en amont et études du comportement des clients fournissent au laboratoire central d'Ikea des informations pour concevoir les produits. Est ensuite fixé le prix de vente en fonction de la concurrence et du degré d'innovation du produit. 34 bureaux d'achat, répartis dans le monde entier et mis en compétition entre eux, vont alors prospecter les fournisseurs. L'entreprise se comporte donc en donneur d'ordre qui fait appel à des sous-traitants auxquels elle impose les spécifications (techniques et de prix) des produits. Enfin, bien sûr, elle joue son rôle central de distributeur. IKEA produit de manière autonome $10 \%$ de ses articles et exploite ses propres scieries et fabriques, réunies dans le Groupe Swedwood. Le groupe peut ainsi garantir une capacité de production à long terme sur des articles de base. Les bureaux d'achat se chargent d'acquérir les $90 \%$ restant auprès de fournisseurs externes. Certains travaillent avec le groupe depuis le tout début, d'autres ont des usines en Chine, en Pologne ou en Allemagne. IKEA est donc une entreprise mondialisée et intégrée avec 1220 fournisseurs dans 55 pays, dont un tiers environ se trouvent en Asie. 
Figure 3 : Gouvernance et réseau de distribution chez IKEA

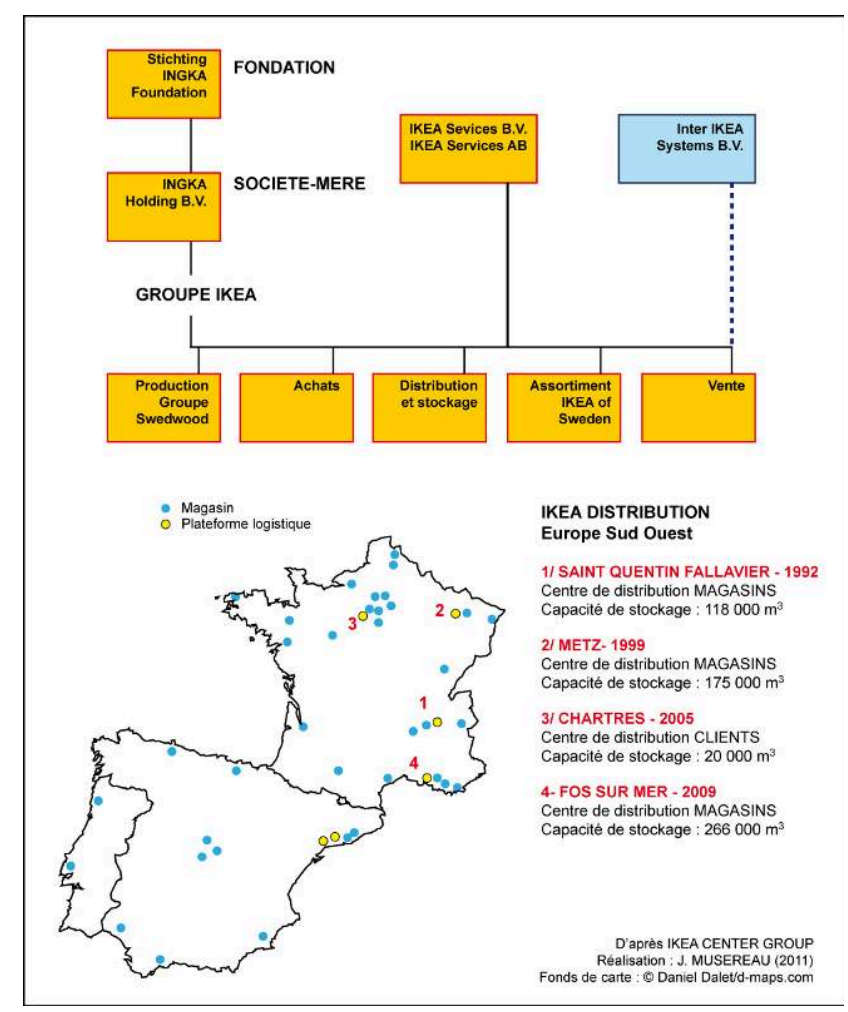

Comme la plupart des gros distributeurs, dès son origine, IKEA a tenté d'acquérir les terrains autour de tous ses magasins. Parallèlement, la création de la filiale Inter Ikea Centre Group lui a permis de passer de simple loueur à gestionnaire de centres. La carte européenne d'Inter Ikea Centre ne cesse d'ailleurs d'évoluer. De 24 centres déclarés ouverts au mois de mai 2010, le groupe est passé à 28 en juin. Quant aux complexes en développement, ils sont actuellement 23, en France, au Portugal, en Croatie ou encore en Chine. En les réalisant, la société doublera sa surface commerciale, qui dépasse déjà le million de mètres carrés, sans compter, dans la famille élargie, les 4 centres gérés par Ikano en Scandinavie et les 12 que détient Ikea Mos en Russie. Comme le relève Angelika Gröschl (Property Manager d'Inter Ikea Centre pour la Suisse), les marchés d'Europe de l'Est, d'ex-Yougoslavie et de Chine sont particulièrement prometteurs : " En 2012, nous allons ouvrir un immense centre à Zagreb, avec plus de 300 enseignes et un magasin Ikea intégré. Pour des développeurs de centres comme nous, Ikea ouvre des marchés où nous n'irions jamais seuls $»^{5}$. Inter Ikea Centre n'est donc qu'une adaptation logique au monde du commerce de détail, où la concentration prime. Le concept outlet, qui consiste à vendre des vêtements de marque 20 à $30 \%$ moins cher en moyenne qu'en magasin, s'est par exemple imposé en Suisse. Cette stratégie induit cependant un effet pervers et très épineux pour une enseigne aussi sensible aux emprises foncières : la saturation des accès au multi-complexe et l'engorgement du parking.

\section{B. La firme nouveau moteur de la requalification des territoires}

Il y a cinq ans, à peine $50 \%$ de la population française était à moins d'une heure d'un IKEA, aujourd'hui c'est $60 \%$. L'objectif est d'atteindre les $80 \%{ }^{6}$. IKEA compte 26 points de vente avec une surreprésentation en Ile-de-France, sur la Côte d'Azur (à l'exception de Nice) et dans l'agglomération lilloise. En revanche, des zones vides demeurent, 
notamment dans le centre du pays. En 2009, la firme a continué à resserrer le maillage en ouvrant deux nouveaux magasins en France, l'un à Thillois (Champagne-Ardenne), l'autre à Vedène (Provence-Alpes-Côte d'Azur), créant respectivement 350 et 300 emplois ${ }^{7}$.

A l'origine d'une implantation plus précoce dans des espaces potentiellement moins attractifs pour la firme, on retrouve assez fréquemment l'argument foncier associé à des stratégies de reconversion du tissu urbain. C'est le cas du centre de la France, «l'arc des déprises ", qui demeurait jusqu'à présent en grande partie délaissé par IKEA. Il faut en effet attendre 2009 pour que l'enseigne se décide à étudier la possibilité d'une implantation à Clermont-Ferrand. Le site envisagé permet au groupe d'implanter à la fois un magasin et un ensemble commercial, donnant ainsi à cet espace une véritable attractivité régionale, et même au-delà. La zone de chalandise porte sur un potentiel de 800000 habitants à 1 ho0 de voiture et 1600000 habitants à une $1 \mathrm{~h} 30$. Ce choix exprime la volonté métropolitaine des élus du grand Clermont d'accroître l'attractivité du territoire par l'arrivée de grandes enseignes internationales. Le site retenu est celui des Gravanches. Il constitue, à proximité du pôle de ré-urbanisation de la friche de l'exusine MSD-Chibret, une réelle opportunité et permet à IKEA d'incarner le rôle d'une véritable "locomotive régionale " (terminologie utilisé dans la campagne publicitaire relative au projet). Cette nouvelle zone aurait ainsi vocation à devenir le principal animateur d'un pôle stratégique d'urbanisme commercial dans un espace déprimé. A ce stade, IKEA reflète bien l'organisation d'une entreprise actrice dont le comportement repose "sur des considérations techniques, politiques, stratégiques [...], ce qui l'amène à privilégier des espaces indépendamment de leur position hiérarchique ou de leurs fonctions économiques» (Fache, 2008).

\section{Libres négociations et indispensables partenaires}

«La place d'une grande ville passe par un Ikéa, un multiplexe et une équipe de foot en ligue 1!» affirme François Cuillandre, maire de Brest $^{8}$ et Président de la communauté urbaine Brest Métropole Océane (BMO). Le magasin IKEA à Brest-Guipavas se situe au cœur de la zone du Froutven, sur un terrain de 6 hectares. La surface commerciale s'étend sur 17 $650 \mathrm{~m}^{2}$, un peu plus qu'à Nantes-Saint-Herblain. Près du grand magasin, une importante zone d'activité de $37000 \mathrm{~m}^{2}$ vise à structurer la partie est de l'agglomération Brestoise. Un très vaste Décathlon de $9000 \mathrm{~m}^{2}-$ le $7^{\text {ème }}$ plus vaste au monde - ainsi qu'une grande enseigne d'électroménager, un magasin Jardiland, divers commerces restaurants et au total une dizaine d'enseignes nationales, spécialisées dans l'équipement et la décoration de la maison. L'investissement global s'élève à 30 millions d'euros et s'intègre dans un concept de retail park initié en France à Hénin-Beaumont Maison plus - autour de la firme suédoise. L'objectif est de profiter d'un effet levier pour développer une nouvelle zone commerciale à l'entrée de la ville, «les Portes de Brest-Guipavas » et de repositionner l'agglomération brestoise face au risque d'évasion commerciale de la métropole vers Rennes et Nantes9.

La zone de chalandise est vaste et se situe à l'ouest d'une ligne Guingamp-Lorient. Ce retail park est l'un des premiers de France (comme celui du Mans). Le projet immobilier est adossé à un chantier public relayé par les acteurs locaux qui prévoient de relier cet espace au reste de la vielle via la ligne de tramway et de renforcer l'identification ludique de la nouvelle zone en y implantant un grand stade de football et un Zénith. La visée métropolitaine est clairement affichée par le maire de la ville considérant 
l'arrivée d'IKEA comme un levier à l'action publique que la notoriété de l'enseigne et les dividendes économiques permettent d'impulser. François Cuillandre souhaite souligner l'envergure du programme en pensant l'attractivité du site à une échelle plus vaste encore : « Avec Ikea et un ensemble d'enseignes de renom, ce nouvel espace commercial s'inscrit complètement dans cette démarche d'attractivité et de rayonnement de notre agglomération ${ }^{10}$. Cette ambition est notamment rendue possible par la conduite d'un projet mixte, piloté par Altarea ${ }^{11}$ (porteuse de la maitrise d'ouvrage), et par l'effet d'entrainement de l'offre commerciale générée par la firme suédoise. A l'initiative de multiples projets ${ }^{12}$, la municipalité assume, au-delà des controverses internes comme externes au projet, un « portage communicationnel» (Barthel, 2007) des aménagements autour de la mise en place du retail park. Mais jusqu'où peut aller le portage politique ?

\section{La firme transnationale face au millefeuille territorial français. Les ressorts de la transaction}

A la fois catalyseur et moteur de nouvelles dynamiques, le pionnier de l'ameublement low-cost se présente comme un acteur à part entière d'une construction territoriale négociée. Certaines firmes ont participé de manière emblématique à la reconnaissance et la qualification de leur espace d'accueil, comme Jalabert (1995) a pu le démonter dans le cas de l'industrie aéronautique à Toulouse. Il est en revanche mois fréquent d'observer une forme de (re)qualification territoriale par les pratiques de consommation (Andersson, 2009). Mais peut-on réellement considérer l'investissement du géant suédois comme la pierre angulaire du développement régional en ancrant localement une dynamique de projet chez les acteurs publics?

\section{A. Conflits d'intérêt et négociations : «à la recherche d'un compromis institutionnalisé ${ }^{13}$.}

La firme ne provoque pas toujours les effets de relance suggérés par ses représentants. Elle contribue auprès des acteurs locaux à créer les conditions et les facteurs qui lui sont nécessaires en façonnant l'agencement des territoires qu'elle investit (Fache, 2008). Face à l'emprise foncière et aux réaménagements des lieux, des fronts de protestations émanant des riverains s'adjoignent aux réserves des commissions ad hoc. À Mougins, la Commission Départementale d'Equipement Commercial (CDEC) a rejeté en 2007 l'autorisation d'implantation d'un magasin IKEA. À Hénin-Beaumont, la CDEC, puis la CNEC, ont également opposé leur refus en janvier 2006. On retrouve un schéma similaire à Montpellier, où l'accord de la CDEC a été cassé par le Tribunal administratif après ouverture du magasin. À Brest, une procédure en référé a été engagée contre l'accord de la CDEC et le magasin a ouvert sans CDEC valable.Le déroulement de l'installation d'IKEA dans les Alpes-maritimes est représentatif des conflits d'intérêt qui peuvent être localement générés par la firme. Après différents épisodes, ce projet d'implantation s'est finalement soldé par une victoire du géant du meuble. Les trois commissaires chargés de mener l'enquête publique pour l'implantation de l'espace commercial "Family Village" à Mougins (figure 4), ont rendu leurs premières conclusions favorables. La commission départementale d'équipement commercial a également validé le projet en l'état. La CDEC l'a en revanche retoqué. Celle-ci ayant par ailleurs soumis une multitude de propositions d'amélioration au projet : l'allongement 
des bretelles de la sortie d'autoroute, la création de passage inférieur au niveau du rond-point du Tiragon, le raccordement du site à la PCG (pénétrante Cannes-Grasse) par le Sud et non pas par la sortie existante, ainsi que la création d'un accès différent pour le pôle et les activités déjà existantes sur le site, et enfin l'aménagement à 3 voies de la RD409. D'importants travaux et aménagements s'imposaient donc avant toute installation du « Family Village », pour un surcoût total de plus de 16 millions d'euros.

Figure 4 : Aménagements requis pour le complexe IKEA à Mougins

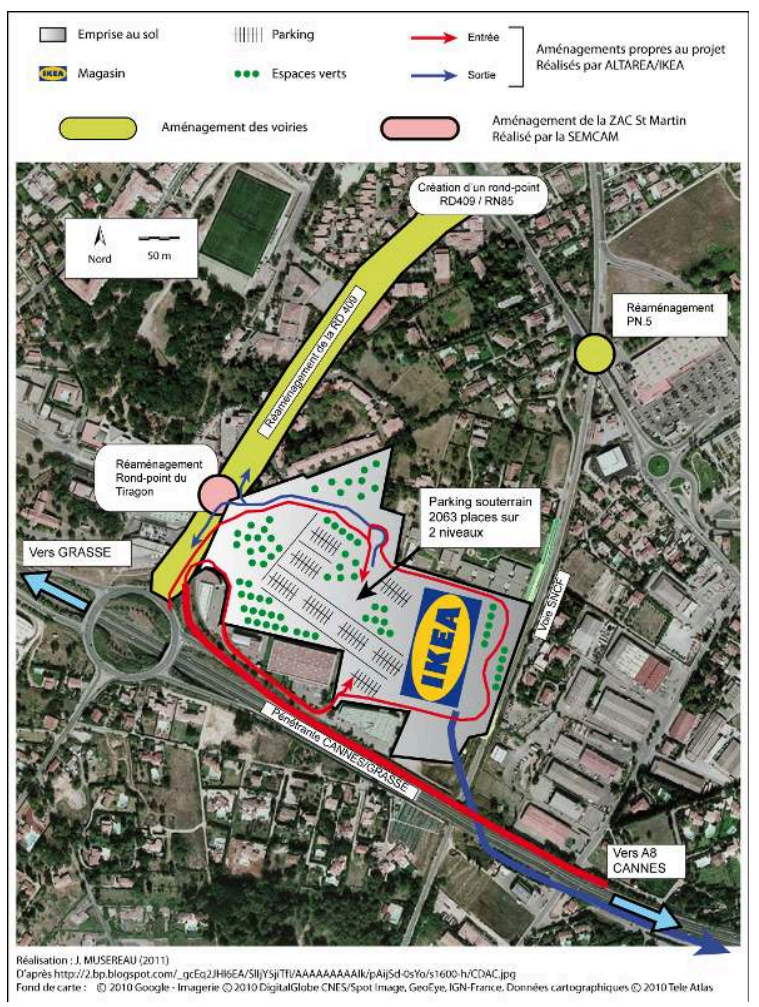

Si la fin des années 1980 s'était faite porteuse d'interrogations en direction d'un assouplissement de la loi Royer et de l'aménagement commercial, le législateur n'a pas modifié les compétences fondamentales des communes (permis de construire), ni le rôle des commissions départementales qui évaluent le bien fondé de la création du commerce si celui-ci est supérieur aux seuils de la loi Royer ${ }^{14}$. D'un point de vue économique, les collectivités qui cherchent à bénéficier d'une dynamique et de ressources leur permettant de palier aux nouvelles compétences qui leur sont dévolues voient leur marge de manœuvre se réduire (exit la taxe professionnelle). Le schéma de Michel Senelet (1987), selon lequel la grande distribution serait capable de financer la réurbanisation des centres villes, semble encore inabouti. Le groupe suédois fonctionne à l'évidence comme une structure tremplin, un point d'attraction régional mais, en définitive, il ne permet pas d'aménagement - civiquement - raisonné des lieux. Dans certains cas, les territoires semblent même souffrir d'un nouvel effet tunnel.

\section{B. Quelle maîtrise politique du territoire ?}

La complexité de la régulation et de la construction de projets territoriaux révèle une revalorisation de la sphère et des déterminants locaux. Plus qu'une totale (ou réelle) réémergence du local, elle souligne en premier lieu le rôle prépondérant de la 
coopération territoriale et du jeu des acteurs. Si, "pour attirer les investisseurs, les autorités locales doivent agir comme un acteur collectif» (Lefevre, 2009), la coopération et les négociations des acteurs locaux sur les conditions d'accueil de la firme restent l'occasion de lire les enjeux politiques des dimensions économiques de l'aménagement du territoire.

Lorsqu'IKEA manifeste en 1984 son intérêt pour l'agglomération rennaise, les élus n'y voient pas immédiatement, ni unanimement, un atout pour le développement local. Le dossier mobilise peu. C'est finalement la conjonction entre un remaniement administratif et politique et la persévérance du groupe suédois qui permet l'ouverture effective du magasin... 24 ans plus tard! Au long de cette période, le jeu des acteurs politiques locaux s'est progressivement organisé et structuré. Les collectivités territoriales ont vu leur rôle s'accroître. Le poids des relations horizontales entre les différents partenaires est devenu une charnière de l'action publique (Pinson, 2010). Les cadres de régulation de l'action publique se sont ouverts à une multitude d'acteurs ouvrant la porte à des institutions mixtes à l'image des SEM. Le projet d'implanter un magasin au sein de la ZAC Les Touches rebaptisée pour l'occasion "Rive Ouest», commune de Pacé (figure 5), soulève un ensemble de questionnements qui vont se révéler essentiellement techniques mais surtout indicatifs des jeux de partenariat et de coalition d'intérêt à l'échelle locale. Malgré des sensibilités politiques divergentes entre la commune d'accueil et Rennes Métropole, aucun antagonisme réellement politique n'est venu freiner le dossier. En revanche le nœud gordien est apparu là où les acteurs ne l'attendaient pas, entre l'ensemble des acteurs locaux et les services étatiques. Parmi les principaux acteurs: Pacé, la commune d'accueil, la communauté d'agglomération Rennes Métropole à laquelle elle adhère, le Département d'Ille-et-Vilaine et l'Etat, la CDEC, la préfecture, les services de la DDE et les sociétés d'économie mixte (SEMAEB et Territoires), Cora-Foncière propriétaire d'une partie de la ZAC ainsi que de manière discrète mais constante IKEA. Le terme de coalition, couramment utilisé au regard de la théorie des régimes urbains, permet d'intégrer la porosité des processus de recomposition des gouvernements et de contrôle des territoires. L'objectif de ces coalitions serait ainsi de tendre vers « des coopérations orientées vers la structuration d'une capacité d'action collective opératoire » (Dormois \& Menez, 2007). 
Figure 5 : Implantation d'IKEA à Pacé

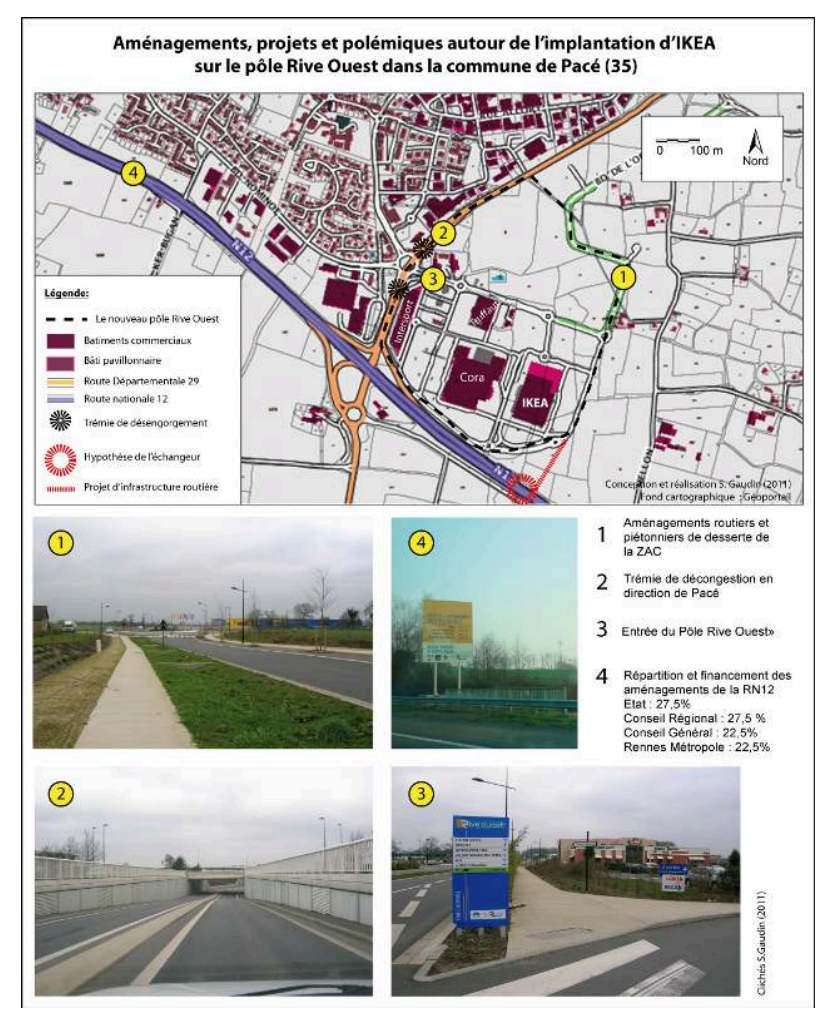

Pour le site rennais, la difficulté a porté sur l'enjeu des infrastructures routières, la liaison du site et son raccordement à la fois aux routes nationale, départementales et communales. L'ampleur du trafic généré par l'installation d'IKEA risquait de faire peser sur la collectivité une partie du coût de ces aménagements. Progressivement le débat s'est ciblé sur la nature des modifications à réaliser et de facto sur les institutions concernées par leur financement. Nouvel échangeur sur la nationale, trémie de désengorgement locale ou communautaire, le débat fait rage pour s'achever quelques mois avant l'ouverture du site par un arbitrage du préfet en faveur d'une option intermédiaire supportée proportionnellement par les parties. L'accord a abouti, deux trémies seront réalisées en direction de Pacé, sur le site de la ZAC, et une autre au niveau de la RN12, la commune étant particulièrement motivée pour soutenir l'installation d'IKEA. Localement, les acteurs politiques ont conservé une maîtrise forte du dossier contre à la fois le pouvoir central et ses services déconcentrés mais aussi face aux doléances de la firme suédoise. Ce discours affiché par les responsables du projet et notamment la SEM qui réalisait la maitrise d'ouvrage est cependant à relativiser. Les élus ont tenté essentiellement de faire jouer la coalition d'intérêts nouée entre la communauté d'accueil, la communauté d'agglomération et la SEM associée - afin de faire coïncider les objectifs et intérêts locaux avec les attentes de la firme mais tout en maintenant un volontarisme politique assumé de contrôle de leur territoire. Cette volonté modérée mais cadrée est visible à travers la charte d'urbanisme commercial de Rennes ${ }^{15}$ - intégrée au SCOT - qui souligne qu'« Ikéa est une enseigne structurante, de niveau européen, qui dynamisera le commerce de Rennes-Métropole ». Signée en 2007 entre Rennes Métropole et ses partenaires EPCI, Chambre de commerce et d'industrie, Chambre des métiers et de l'artisanat, Union du Commerce et Maison de la Consommation et de l'Environnement, elle affirme le caractère structuré et abouti de la coalition et le rôle de Rennes Métropole comme un espace de régulation économique 
et social. Encouragés par une tentative de gouvernement à distance de l'Etat (Epstein, 2009), les acteurs privés représentent des pivots capables autant de participer à l'émancipation qu'à la stabilisation ou la précarisation des territoires assermentés. Ce qui amène Glin (2009) à conclure sur la prééminence d'une coalition d'acteurs locaux et tend à souligner une certaine fragilité du jeu politique local cerné entre les différents niveaux du mille feuille territorial, d'une part, et la compétition territoriale, d'autre part. A Rennes, la collectivité ne pouvait assumer seule la charge du projet et le débat s'est reporté sur le coût des infrastructures routières incombant en partie à l'Etat et la DDE, un investissement à charge sans retour sur les bénéfices potentiels. Autour des enjeux liés à l'implantation de la firme, IKEA révèle ainsi l'imperfection du transfert des compétences et des attributions financières. Les interactions analysées au travers de cet exemple mettent également en exergue les dissensions entre les différentes strates administratives et politiques, qui reflètent à leur tour le paradoxe d'une décentralisation inaboutie.

\section{Conclusion}

En s'appuyant sur une relecture de la théorie du pouvoir de négociation (Combe \& Mucchielli, 2008), l'objet de cette contribution a été d'analyser la capacité de la multinationale suédoise à interagir avec les pouvoirs locaux en imposant des modèles territoriaux basés sur une concentration raisonnée, une accessibilité contrôlée et une gouvernance partenarisée. A travers l'analyse des stratégies de la firme suédoise dans ses différentes composantes il semble que malgré la partenarisation avancée de certains projets et la cristallisation du jeu des acteurs (public/privé) il n'émerge pas réellement - encore ? - «d'espaces de coordination localisés » selon l'expression de Raveyre (2005). L'implantation de magasins IKEA répond à une rencontre entre intérêts économiques croisés, la reconnaissance métropolitaine d'un système régional et appui d'un projet politique local. Parfois pilote (Clermont-Ferrand, Pacé, Marseille), d'autrefois enclume (Mongins, Hénin-Beaumont) la firme bleu et jaune représente la charnière de nouvelles formes de gouvernance voire d'ingénierie territoriale, emblème de territoires moins touchés par la mondialisation que par la nécessaire imbrication des échelles de décisions et de projets et par de nouvelles formes d'interspatialité.

\section{BIBLIOGRAPHIE}

Andersson F., 2009, Performing Co-production: On the logic and practice of shopping at IKEA, PhD Thesis, Social and Economic Geography, Uppsala University, 200 p.

Andreff W., 1999, «Peut-on empêcher la surenchère des politiques d'attractivité à l'égard des firmes multinationales? », in Bouët A. et Le Chacheux E., Globalisation et politiques économiques : les marges de manœuvre, Paris, Economica, p. 401-424. 
Badré D., Ferrand L., 2001, Mondialisation : réagir ou subir ? La France face à l'expatriation des compétences, des capitaux et des entreprises, Rapports du Sénat, nº 386, Paris, 401 p.

Bailly O., Lambert D. et Caudron J.M., 2006, Ikea, un modèle à démonter, Bruxelles, Editions Luc Pire, $108 \mathrm{p}$.

Barthel P.A., 2007, Renouvellement urbain à Brest : les enseignements du marché de définition du plateau des Capucins, Norois, ${ }^{\circ}$ 203, p. 7-25.

Berthaud P., Rocca M., 2000, « Les relations mondialisation-travail: panorama des approches théoriques », Economies et Sociétés (série Mondialisation et travail), n³7, p.51-86.

Boyer R. 1996, La théorie de la régulation, une analyse critique, Paris, la Découverte, 142 p.

Boyer R., Saillard Y., 1995, Théorie de la régulation : l'état des savoirs, Paris, La Découverte, 568 p.

Cohen E., Lorenzi J-H., 2000, « Des politiques industrielles aux politiques de compétitivité en Europe », in Politiques industrielles pour l'Europe, Rapport du CAE, $n^{\circ} 26$, Paris, La Documentation française, p. 9-172.

Combe E., Mucchielli J.-L., 2008, « The Multinational Firm Versus the Host Country: a Bargaining Power Approach », in Mucchielli J.-L., Research in Global Strategic Management: Multinational Location Strategy, Jai Press, p. 185-210.

Commaille J., Jobert B., 1999, Les métamorphoses de la régulation politique, Paris, LGDJ, 386 p.

Déjardins M., 2002, Stratégie d'un groupe de distribution, IKEA, Mémoire $3^{\text {ième }}$ cycle MLD Marketing \& Distribution, Paris, 21 p.

Dormois R., Menez F., 2007, Renouveler le modèle de développement économique local au nom de l'urbain ? Recherche comparative sur trois villes en reconversion de l'Europe de l'Ouest : Gênes, Saint-Etienne et Sheffield, Paris, PUCA, 123 p.

Epstein R., 2009, « Après la territorialisation, le gouvernement à distance », in Vanier M., Territoire, territorialité, territorialisation : controverses et perspectives, Rennes, Presses Universitaires de Rennes, p. 131-139.

Fache J., 2008, « Quand l'entreprise recompose l'espace acentral », Géographie, économie, société, vol.10, p. 469-492.

Gaudin S., Musereau J., 2009, «IKEA revisite les classiques de la géographie », EspacesTemps.net, Mensuelles, mis en ligne le 26 novembre 2009, consulté le 31 janvier 2011, URL : http:// espacestemps.net/document7953.html

Glin L., 2009, Aménagement du territoire et gouvernance multi-niveaux : coalitions, conflits, régulation. L'implantation d'Ikéa dans l'agglomération rennaise dans un contexte d'emboitement des territoires. Mémoire réalisé dans le cadre du Séminaire Action publique locale, Rennes, Université Rennes 1, IEP, $98 \mathrm{p}$.

Gumuchian H., Grasset E., Lajarge R., Roux E., 2003, Les acteurs, ces oubliés du territoire, Paris, Anthropos/Economica, $186 \mathrm{p}$.

Jalabert G., 1995, Toulouse, métropole incomplète, Paris, Anthropos/Economica, 202 p.

Laffont J-J., Tirole J., 1993, A theory of incentives in procurement and regulation, Cambridge and London, The MIT Press, $731 \mathrm{p}$.

Lefevre P., 2009, Métropolisation et gouvernance urbaine: les dynamiques territoriales du nouveau régionalisme dans les agglomérations de Los Angeles et San Francisco, Thèse de Doctorat, Université Toulouse II le Mirail, 364 p. 
Lorenzi J-H., 2004, « Mondialisation et nouvelle stratégie d'entreprise », in Cercle des économistes, Les entreprises européennes dans la compétition mondiale, Paris, Descartes, p. 152-169.

Norman R., Ramirez R., 1993, «From value chain to value constellation: designing interactive strategy », Harvard Business Review, vol. 71, n 4, p. 65-77.

Pinson G. 2010, « La gouvernance des villes françaises. Du schéma centre-périphérie aux régimes urbains ", Pôle Sud, 2010/1, n 32, p. 73-92.

Porter M.E., 1982, Choix stratégiques et concurrence, Paris, Economica, 426 p.

Raveyre M., 2005, « Restructurations, grands groupes et territoires De l'utilité de la construction d'espaces de coordination localisés ", Géographie Économie Société, 2005/4, vol. 7, p. 333-346.

Rogowski R., 1989, Commerce and coalitions: How Trade Affects Domestic Political Alignments, Princeton, Princeton University Press, 208 p.

Senelet M., 1987, «La planification de l'urbanisme commercial lyonnais », Revue Géographique de Lyon, vol 64, $\mathrm{n}^{\circ} 2$, p.79-86.

Storaï C., 2003, «Attractivité territoriale et stratégies de localisation des firmes : le territoire Corse peut-il devenir un nouvel Eden? ", ISDM, vol. 9, $\mathrm{n}^{\circ} 75$, mis en ligne en juillet 2003, consulté le 31 janvier 2011, URL : http://isdm.univ-tln.fr/PDF/isdm9/isdm9a75_storai.pdf

\section{NOTES}

1. Produit de consommation non courante ni quotidienne, à l'exemple du mobilier et de l'ameublement.

2. «Un Ikea sinon rien!", dossier «Ikea : la secte mondiale du kit », Courrier international, $\mathrm{n}^{\circ} 722$, Paris, 2-8 septembre 2004.

3. Une dénomination en référence non pas à la place jouée par la multinationale suédoise dans les négociations mais aux difficultés du groupe à s'implanter dans la région. Or il est clair que cette loi, même régionalisée en 2009, occulte qu'aujourd'hui les enjeux économiques dépassent largement le cadre territorial de la commune. Une inadéquation soulignée par les instances européennes qui en demandent la révision en janvier 2010.

4. Qualifie l'acte qui vise à réduire le coût général des achats, en automatisant les processus concernés. Cette expression (recherche d'une source) désigne l'action de recherche, de localisation et d'évaluation d'un fournisseur ad hoc.

5. Inter IKEA Group Center - Dossier de presse 2011.

6. Extrait de Dromard T., 2009, "IKEA s'inscrit sur de nouveaux territoires", Challenges, 07.05.2009. Textuel URL : http://www.challenges.fr/magazine/strategie/0167.026527/

7. Bilan 2009 des investissements internationaux en France, AFII, Bercy, 9 mars 2009, 14 p. URL: http://www.invest-in-france.org/Medias/Publications/982/Bilan-investissements-etrangerscreateurs-d'emplois-en-france-2009_FR.pdf

8. Interview sur Breizh-izel, vendredi 7 mai 2010.

9. Avant l'ouverture des sites brestois et rennais, les Brestois représentaient $6 \%$ du chiffre d'affaire du magasin nantais (communiqué IKEA datant du 27 octobre 2007).

10. «IKEA arrive à Brest », 2007, Journal d'information de Brest Guipavas, Le magazine Retail Park, $\mathrm{n}^{\circ} 1$, octobre, p. 2.

11. A Brest, Altarea est également propriétaire de deux grands centres commerciaux : Coat Ar Gueven et l'Espace Jaurès.

12. Brest oriente sa communication territoriale autour d'un nouveau visage de la ville. Ces transformations se lisent également dans les multiples projets en cours (une opération de 
rénovation urbaine multi-site conventionnée par l'ANRU de grande ampleur ; la reconversion du plateau des Capucins, ancienne friche militaire; la mise en place d'une ligne de tramway; l'aménagement d'un mail) et par toute une stratégie métropolitaine revendiquée avec le renforcement de ses pôles de compétitivité ainsi que le renfoncement des infrastructures culturelles de dimension régionale.

13. Boyer \& Saillard (1995), référence complète en bibliographie.

14. En 1993, la loi Sapin réforme le dispositif mis en place par la loi Royer et le gouvernement gèle provisoirement les autorisations d'ouverture. En 1996, la loi Raffarin, qui s'accompagne également d'un gel des ouvertures, abaisse à 300 mètres carrés le seuil à partir duquel une demande d'autorisation est obligatoire.

15. http://www.rennes.cci.fr/wpFichiers/1/1/Telechargements/79/charte-urbanismecommercial-rennes.pdf

\section{RÉSUMÉS}

Malgré une récente inflexion, dans l'ensemble des pays industrialisés, la logique d'attraction des IDE s'est largement substituée aux politiques étatiques de restriction-réglementation des implantations des firmes étrangères (Andreff, 1999). Désormais, ce sont majoritairement les choix stratégiques de localisation des firmes multinationales qui préfigurent la réalisation des nouveaux relais de la croissance en tant qu'élément moteur de la rationalisation de l'économie et de l'agencement des territoires (Cohen \& Lorenzi, 2000). En s'appuyant sur une relecture de la théorie du pouvoir de négociation (Combe \& Mucchielli, 2008), l'objet de cet article consiste à analyser la capacité d'une multinationale suédoise (IKEA) à interagir avec les pouvoirs locaux en imposant des modèles territoriaux basés sur une concentration raisonnée, une accessibilité contrôlée et une gouvernance partenarisée. Mais qu'apporte l'implantation d'un IKEA au territoire local ? Quelles sont les implications d'un groupe à la stratégie mondiale et mondialisée dans les perspectives d'un développement local?

Logic of FID attraction has been progressively substituted to the former strategies that were developed by states regulatory policies and planning bodies (Andreff, 1999). From now on, companies' location strategy is commonly related to economic growth, as it constitutes an element of rationalization of the economy and of territory organization (Cohen \& Mucchielli, 2000). Many theories were developed to better understand the implicated mechanisms, as the "Bargaining Power Approach" of Combe \& Mucchielli (2008). With the example of IKEA, this study aims at offering a new interpretation of this work. Using a multi-scaling approach, and then, focusing on complex interactions between the swedish company and local authorities, we point out how firms are able to lay down political rescaling, notably through the argument of concerted governance. However, recent and controversial implantations incite us to moderate such an assertion. Is it still indisputably beneficial to welcome an IKEA at home?

In den Industriestaaten hat die Anziehungslogik der internationalen Direktinvestitionen die auf Beschränkung und Regelung basierte Politik ersetzt, welche die Staaten durchsetzten, wenn eine ausländische Firma sich ansiedelte (Andreff, 1999). Von nun an sind die Lokalisierungsstrategien der multinationalen Unternehmen mit der Förderung des Wachstums verbunden, sie fördern auch die Rationalisierung der Wirtschaft und der Raumordnung (Cohen \& Lorenzi, 2000). Auf 
einer Neuinterpretation der Theorie der Verhandlungsmacht (Combe \& Mucchielli, 2008) basierend, analysiert dieser Aufsatz die Fähigkeit eines schwedischen multinationalen Unternehmens (IKEA), mit den Ortsbehörden $\mathrm{zu}$ verhandeln und territoriale Modelle durchzusetzen, die auf einer durchdachten Konzentrierung, einer kontrollierten Zugänglichkeit und einer geteilten Verwaltung beruhen. Die Beispiele für die Hegemonie des schwedischen Riesen sind zahlreich. Studiert man aber neue oder umstrittene Fälle, sieht man, dass das IKEAModell bei seinen Erneuerungsversuchen auf Schwierigkeiten stößt. Ist es immer noch ein Vorteil, ein IKEA in seiner Nähe zu haben?

\section{INDEX}

Keywords : bargaining, IKEA, local power, location strategies, transnational firms, urban politics Schlüsselwörter : Ansiedlungsstrategie, IKEA, Kommunalverwaltung, Stadtpolitik, transnationale Unternehmen, Verhandlung

Mots-clés : firme transnationale, IKEA, négociation, politique urbaine, pouvoirs locaux, stratégies d'implantation

\section{AUTEURS}

\section{JONATHAN MUSEREAU}

Docteur, équipe COSTEL, UMR-6554 LETG CNRS, Université Rennes 2 - Place du Recteur Henri le Moal, 35043 Rennes Cedex, jonathan.musereau@sfr.fr

\section{SOLÈNE GAUDIN}

PRAG, équipe ESO-Rennes, UMR-6590 CNRS, Université Rennes 2 - Place du Recteur Henri le Moal, 35043 Rennes Cedex, solene.gaudin@uhb.fr 